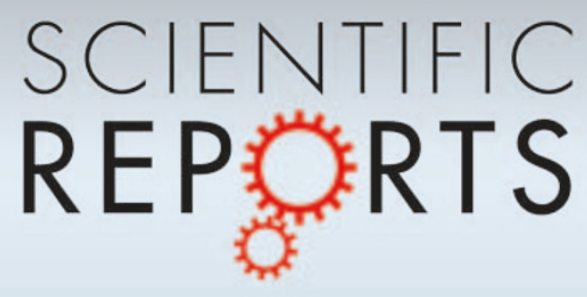

OPEN

SUBJECT AREAS:

APPLIED PHYSICS

MODELLING AND THEORY

MATERIALS PHYSICS

CONDENSED MATTER PHYSICS

Received

19 January 2012

Accepted

10 April 2012

Published

23 April 2012

Correspondence and requests for materials should be addressed to

J.Z.J. (jiangiz@zju. edu.cn)

\title{
Pressure-induced
}

\section{amorphous-to-amorphous configuration change in Ca-Al metallic glasses}

\author{
H. B. Lou', Y. K. Fang', Q. S. Zeng' ', Y. H. Lu', X. D. Wang', Q. P. Cao', K. Yang ', X. H. Yu' ', L. Zheng', \\ Y. D. Zhao ${ }^{3}$, W. S. $\mathrm{Chu}^{3,4}$, T. D. Hu ${ }^{3}$, Z. Y. Wu ${ }^{3,4}$, R. Ahuja ${ }^{1,5,6}$ \& J. Z. Jiang ${ }^{1}$
}

'International Center for New-Structured Materials and Laboratory of New-Structured Materials, State Key Laboratory of Silicon Materials \& Department of Materials Science and Engineering, Zhejiang University, Hangzhou 310027 , P.R. China, ${ }^{2}$ Shanghai Institute of Applied Physics, Chinese Academy of Sciences, Shanghai 201203, P.R. China, ${ }^{3}$ Beijing Synchrotron Radiation Facility, Institute of High Energy Physics, Chinese Academy of Sciences, Beijing, P.R. China, ${ }^{4}$ National Synchrotron Radiation Laboratory, University of Science and Technology of China, Hefei, Anhui 230029, P.R. China, ${ }^{5}$ Condensed Matter Theory Group, Department of Physics, Uppsala University, Uppsala, Sweden, ${ }^{6}$ Department of Materials Science and Engineering, Royal Institute of Technology (KTH), Stockholm, Sweden.

Pressure-induced amorphous-to-amorphous configuration changes in Ca-Al metallic glasses (MGs) were studied by performing in-situ room-temperature high-pressure $\mathrm{x}$-ray diffraction up to about $40 \mathrm{GPa}$. Changes in compressibility at about $18 \mathrm{GPa}, 15.5 \mathrm{GPa}$ and $7.5 \mathrm{GPa}$ during compression are detected in $\mathrm{Ca}_{80} \mathrm{Al}_{20}, \mathrm{Ca}_{72.7} \mathrm{Al}_{27.3}$, and $\mathrm{Ca}_{66.4} \mathrm{Al}_{33.6} \mathrm{MGs}$, respectively, whereas no clear change has been detected in the $\mathrm{Ca}_{50} \mathrm{Al}_{50} \mathrm{MG}$. The transfer of $\mathrm{s}$ electrons into $\mathrm{d}$ orbitals under pressure, reported for the pressure-induced phase transformations in pure polycrystalline $\mathrm{Ca}$, is suggested to explain the observation of an amorphous-to-amorphous configuration change in this Ca-Al MG system. Results presented here show that the pressure induced amorphous-to-amorphous configuration is not limited to felectron-containing MGs.

$\mathrm{T}$

he amorphous-to-amorphous configuration change (AACC) induced by pressure has been a topic of considerable research activities in several substances, e.g., ice, silicon, silica, and carbon ${ }^{1-10}$. Structural polyamorphic transitions from a low-density amorphous state to high-density amorphous state often result in an increase in atomic coordination. Such coordination increase, and thus polyamorphism, was thought to be impossible in non-directional, densely-packed metallic glasses (MGs), characterized by high coordination numbers, e.g., even up to 12-14 random nearest neighbors. Very recently, AACCs were surprisingly observed in Cecontaining MG systems ${ }^{11-17}$, in which the nature of AACCs is revealed due to $4 f$ electron delocalization in Ce under high pressure ${ }^{14}$. Up to now no experimental evidence for AACCs has only been reported in non-f-electroncontaining MGs. An interesting question has been raised: is AACC universal feature in metallic glasses or only in systems having $\mathrm{f}$ electrons? i.e., Can AACC occur in non-f-electron-containing MGs? Here we report pressureinduced amorphous-to-amorphous configuration changes in $\mathrm{Ca}-\mathrm{Al} \mathrm{MGs}$ detected by in-situ room-temperature high-pressure x-ray diffraction (XRD) measurements up to about $40 \mathrm{GPa}$. Changes in compressibility at about $18 \mathrm{GPa}, 15.5 \mathrm{GPa}$ and 7.5 GPa during compression have been detected in $\mathrm{Ca}_{80} \mathrm{Al}_{20}, \mathrm{Ca}_{72.7} \mathrm{Al}_{27.3}$, and $\mathrm{Ca}_{66.4} \mathrm{Al}_{33.6}$ MGs, respectively, whereas no clear change is detected in the $\mathrm{Ca}_{50} \mathrm{Al}_{50} \mathrm{MG}$. Possible mechanism for the AACC is presented and discussed. These results obtained here point out that the amorphous-to-amorphous configuration changes induced by pressure are not limited to f electron-containing MGs.

\section{Results}

In-situ high-pressure XRD patterns for all samples studied were recorded at room temperature up to about $40 \mathrm{GPa}$. No crystallization reaction was detected in these samples in the studied pressure range whereas it was recently reported in the Ce-Al system ${ }^{18,19}$. Figure 1 shows selected high pressure $\mathrm{x}$-ray diffraction patterns during compression for the $\mathrm{Ca}_{80} \mathrm{Al}_{20} \mathrm{MG}$ alloy at room temperature. With increasing pressure, the main amorphous diffraction peak together with sharp Au peaks shift to high $2 \theta$, as expected for the densification effect of pressure. The sample retains fully amorphous structure up to about $40 \mathrm{GPa}$ by judging from the smooth broad patterns. The reverse main amorphous diffraction peak position, $\lambda /(2 \sin \theta)$, correlates with the volume of glass having a power law function ${ }^{20-22}$, which can be conveniently used to reflect the relative volume (density) change as a 


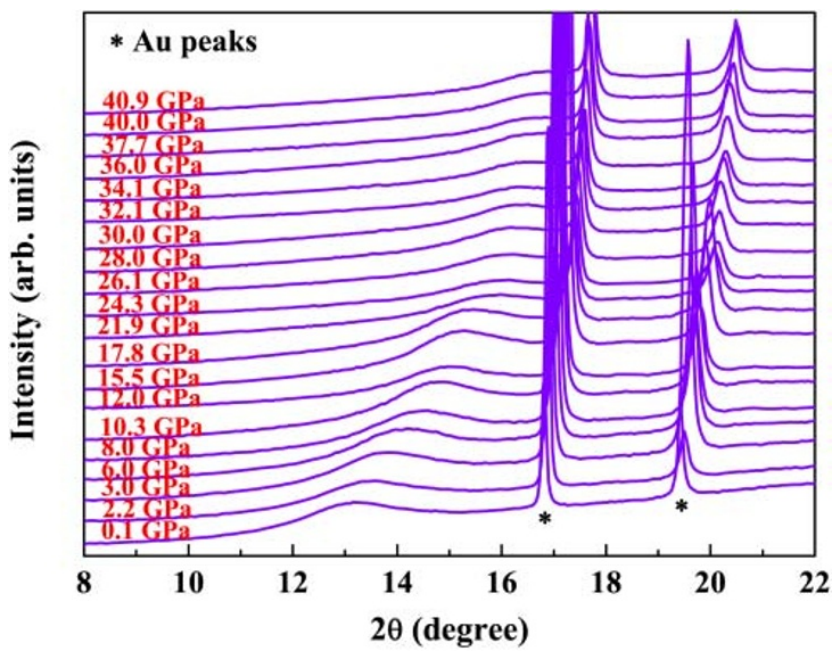

Figure $1 \mid$ In-situ high pressure $x$-ray diffraction patterns of the $\mathrm{Ca}_{80} \mathrm{Al}_{20}$ MG during compression at room temperature from 0.1 to $40.9 \mathrm{GPa}$. The position of the broad amorphous peak shifts to higher angles with increasing pressure. Au peaks as pressure calibrant are marked.

function of pressure. Figure 2 shows the inverse main amorphous diffraction peak position, $\lambda /(2 \sin \theta)$, of the $\mathrm{Ca}_{100-\mathrm{x}} \mathrm{Al}_{\mathrm{x}}(\mathrm{x}=20,27.3$, and 33.6 at.\%) MGs as a function of pressure during compression, which were estimated from the diffraction peak fitting using a Voigt line profile after subtracting baseline. It is found that at about $18 \mathrm{GPa}, 15.5 \mathrm{GPa}$ and $7.5 \mathrm{GPa}$, clear changes were detected for $\mathrm{Ca}_{80} \mathrm{Al}_{20}, \mathrm{Ca}_{72.7} \mathrm{Al}_{27.3}$, and $\mathrm{Ca}_{66.4} \mathrm{Al}_{33.6} \mathrm{MGs}$, respectively, whereas no clear change was detected for the $\mathrm{Ca}_{50} \mathrm{Al}_{50} \mathrm{MG}$. Amorphous-toamorphous configuration changes occur in $\mathrm{Ca}_{80} \mathrm{Al}_{20}, \mathrm{Ca}_{72.7} \mathrm{Al}_{27.3}$, and $\mathrm{Ca}_{66.4} \mathrm{Al}_{33.6} \mathrm{MGs}$ during compression. During pressure releases, it is found that the transformation is reversible with hysteresis. To further support this scenario, we carried out the following considerations.

\section{Discussion}

To uncover the change detected in Fig. 2, it might be useful to revisit the pure polycrystalline $\mathrm{Ca}$. It was reported that for a pure polycrystalline $\mathrm{Ca}$ at ambient temperature, a face center cubic (fcc)-to-body

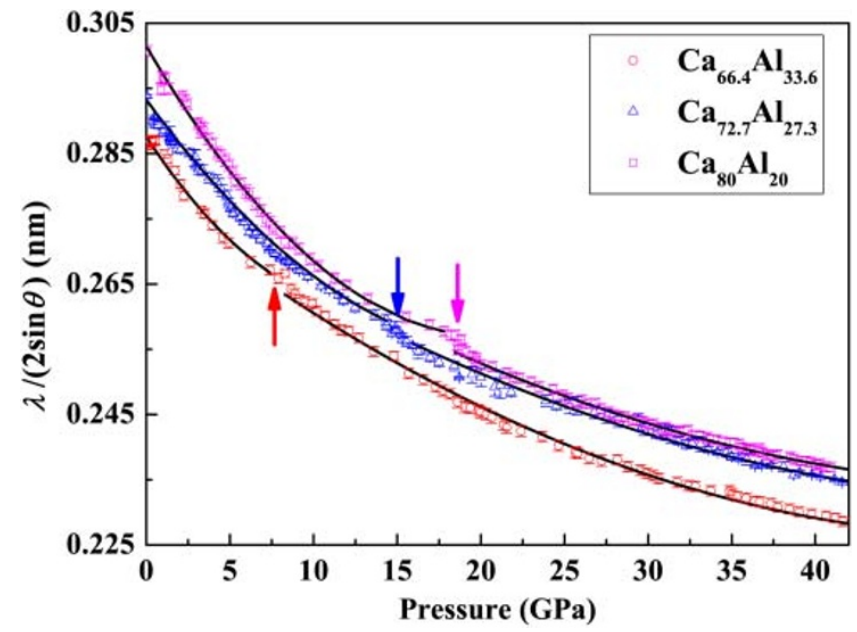

Figure $2 \mid$ The parameter, $\lambda /(2 \sin \theta)$, of the main amorphous peak, which is related to the reduced volume of the sample $\mathrm{V}(\mathrm{P}) / \mathrm{V}(0)$, as a function of pressure for $\mathrm{Ca}_{100-\mathrm{x}} \mathrm{Al}_{\mathrm{x}}(\mathrm{x}=20,27.3$, and 33.6 at.\%) MGs. Transitions are marked by arrows. Error bars for experimental data are given and some are smaller than the symbol size. The solid lines are guides to the eye.
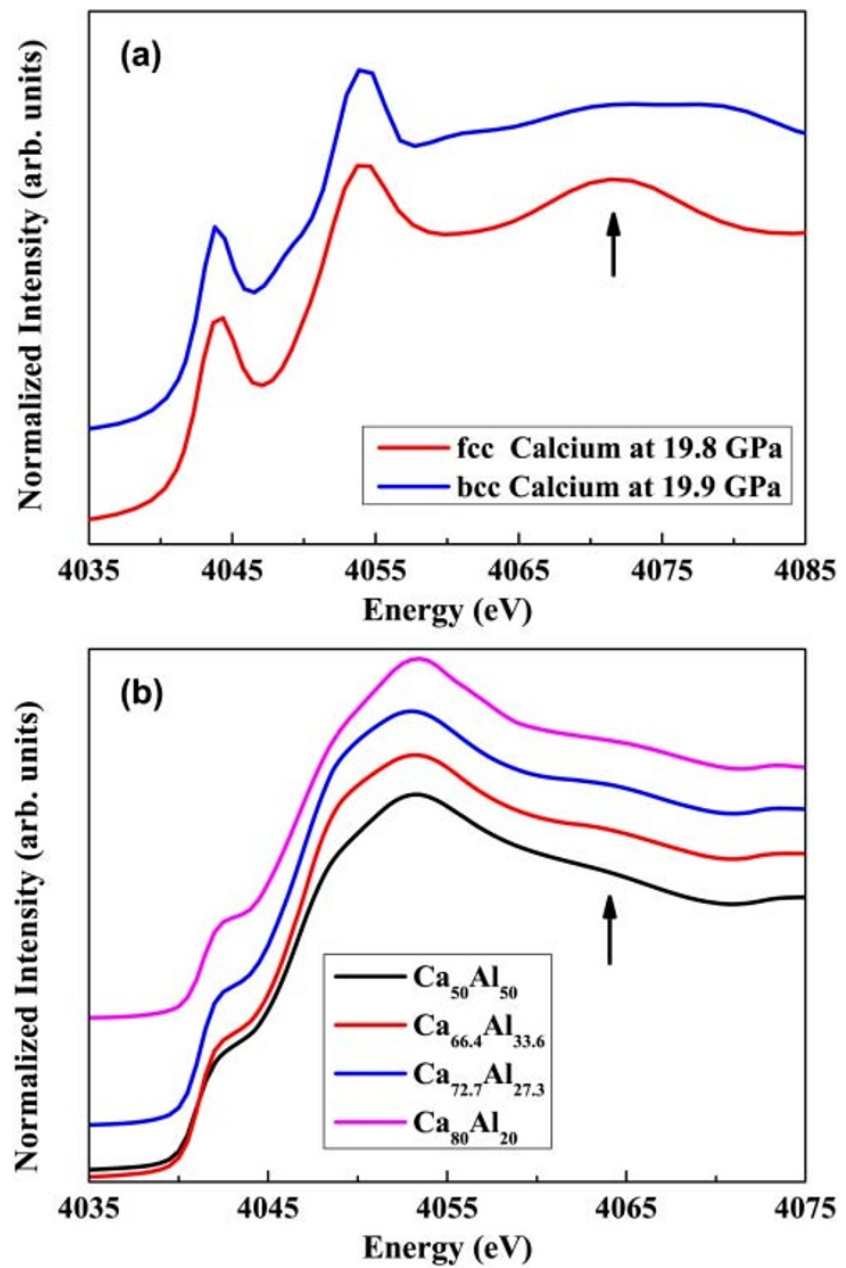

Figure 3 Calculated and experimental data of Ca $K$-edge XANES for pure Calcium and CaAl metallic glasses respectively. (a) K-edge XANES spectra for pure polycrystalline fcc-Ca (at $19.8 \mathrm{GPa}$ ) and bcc-Ca (at $19.9 \mathrm{GPa}$ ) as obtained by calculations in the framework of the multiplescattering (MS) theory using the FEFF 8.2 code. Lattice parameters for both fcc- and bcc-Ca phases are from Ref. ${ }^{23}$ (b) Ca K-edge x-ray absorption near edge structure experimental curves for $\mathrm{Ca}_{100-\mathrm{x}} \mathrm{Al}_{\mathrm{x}}(\mathrm{x}=20,27.3,33.6$ and 50 at.\%) MGs at ambient pressure.

center cubic (bcc) phase transition was detected at 19.8 GPa during compression accompanied by a volume change of about $2-3 \%^{23-30}$, which is much smaller than about $15 \%$ for pure $\mathrm{Ce}^{14}$. This transition was strongly linked with the transfer of s electrons into d orbitals of $\mathrm{Ca}$ under pressure ${ }^{26,28}$. We further performed both fcc- and bcc-Ca $K$-edge x-ray absorption near edge structure (XANES) calculations at 19.8 GPa and 19.9 GPa, respectively, as shown in Fig. 3a. By comparing XANES curves of fcc-Ca with bcc-Ca, the striking feature detected is the intensity change of the peak $(4.07 \mathrm{keV})$ at around $15 \mathrm{eV}$ above the main peak during the transition. We attempted to record XANES data for both $\mathrm{Ca}$ and $\mathrm{Al}$ edges for our Ca-Al MGs under pressures. However, due to extreme experimental difficulties, e.g., high absorption of low edge-energy of both $\mathrm{Ca}$ and $\mathrm{Al}$, experimental XANES data for all studied $\mathrm{Ca}-\mathrm{Al} \mathrm{MGs}$ under pressure were not obtained. On the other hand, we do obtain good Ca $\mathrm{K}$-edge XANES data for our studied $\mathrm{Ca}_{100-\mathrm{x}} \mathrm{Al}_{\mathrm{x}}(\mathrm{x}=20,27.3,33.6$ and 50 at.\%) MGs at ambient pressure, as shown in Fig. 3b. Although the Ca $K$-edge XANES curves for $\mathrm{Ca}_{100-\mathrm{x}} \mathrm{Al}_{\mathrm{x}}(\mathrm{x}=20,27.3,33.6$ and 50 at.\%) MGs differ from that for pure polycrystalline $\mathrm{Ca}$, one similar feature observed is that about $15 \mathrm{eV}$ above main peak a hump is detected for all studied $\mathrm{Ca}-\mathrm{Al} \mathrm{MGs}$. For the $\mathrm{Ca}_{50} \mathrm{Al}_{50} \mathrm{MG}$, relatively speaking, the 


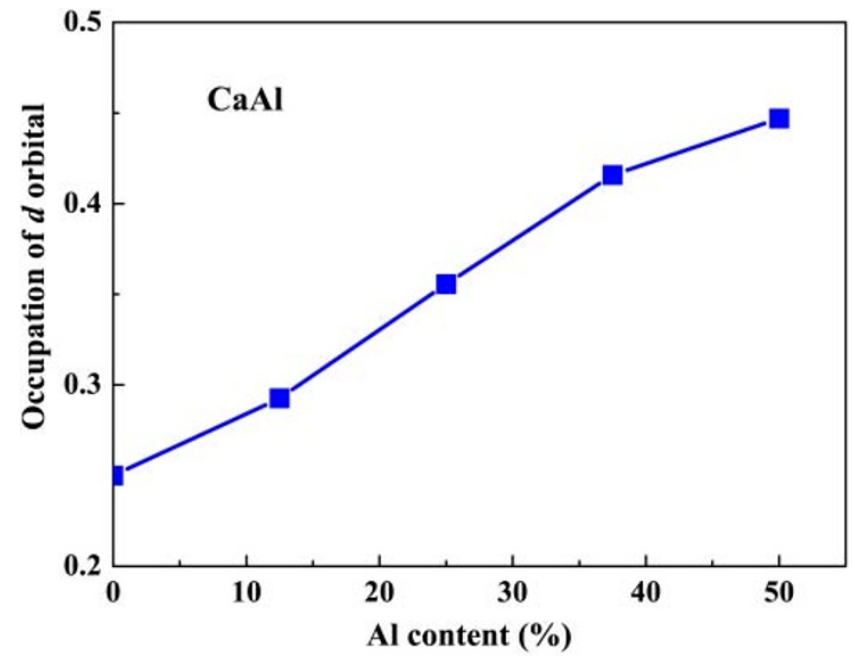

Figure $4 \mid$ Electrons occupying d orbitals of $\mathrm{Ca}$ in the dilute mode for the $\mathrm{Ca}-\mathrm{Al}$ binary system as a function of $\mathrm{Al}$ content obtained from firstprinciples calculations. The occupation of $\mathrm{d}$ orbitals of $\mathrm{Ca}$ increases from 0.25 in pure fcc-Ca to 0.45 when $\mathrm{Al}$ concentration is $50 \%$.

intensity of this hump is obvious low as compared with the other samples. This is also confirmed by further calculated $\mathrm{Ca} \mathrm{K}$-edge XANES data using FEFF code for the $\mathrm{Ca}_{13} \mathrm{Al}_{14}$ and $\mathrm{Ca}_{8} \mathrm{Al}_{3}$ alloys at ambient pressure (see Supplementary Fig. S1 online). To shed light on the Al effect on the charge transfer from $s$ and/or p orbitals to $\mathrm{d}$ orbitals of $\mathrm{Ca}$, we performed first-principles calculations based on density functional theory (DFT) for electronic structures of Ca-Al alloy. We found that $\mathrm{Ca}$ orbitals dominate around the Fermi level even at high $\mathrm{Al}$ concentration up to $50 \%$, although the character of $\mathrm{d}$ orbitals is reduced when $\mathrm{Al}$ concentration increases. $\mathrm{Al}$ atoms provide more electrons occupying $\mathrm{d}$ orbitals of $\mathrm{Ca}$ in the dilute model as showed in Fig. 4. In the pure fcc-Ca system, the occupation of d orbitals is only 0.25 and it increases to $0.29,0.36$ and 0.45 when the concentration of $\mathrm{Al}$ is 12.5 at.\%, 25 at.\% and 50 at.\%, respectively.

Based on all considerations mentioned above, we suggest that the role of $\mathrm{Al}$ in binary $\mathrm{Ca}-\mathrm{Al}$ alloys could be treated as "chemical pressure" to $\mathrm{Ca}$, which promotes the transfer from $\mathrm{s}$ and $\mathrm{p}$ orbitals to $\mathrm{d}$ orbitals of $\mathrm{Ca}$, explaining the experimental fact of lowering the AACC transition pressure from $19.8 \mathrm{GPa}, 18 \mathrm{GPa}, 15.5 \mathrm{GPa}$ to $7.5 \mathrm{GPa}$ as $\mathrm{Al}$ content increases from 0 at.\%, 20 at.\%, 27.3 at.\% to 33.6 at.\%, respectively. A similar role of "chemical pressure" was also reported in the literatures ${ }^{31-35}$. For the $\mathrm{Ca}_{50} \mathrm{Al}_{50} \mathrm{MG}$, following the "chemical pressure" effect, AACC already occurs at ambient pressure because of high occupation of Ca d orbitals, in agreement with the experimental observation: no change during compression in the studied pressure range.

In conclusions, we reported amorphous-to-amorphous configuration changes induced by pressure in non-f-electron-containing $\mathrm{Ca}_{100-\mathrm{x}} \mathrm{Al}_{\mathrm{x}}(\mathrm{x}=20,27.3$ and 33.6 at.\%) metallic glasses, which were confirmed by in-situ room-temperature high-pressure x-ray diffraction up to about $40 \mathrm{GPa}$. By comparing with pure polycrystalline $\mathrm{Ca}$, together with XANES data obtained from both experiments and theoretical calculations, one mechanism, i.e., the charge transfer of $\mathrm{s}$ and $\mathrm{p}$ orbitals to $\mathrm{d}$ orbitals of $\mathrm{Ca}$ under pressure, is suggested for the origin of the amorphous-to-amorphous configuration changes induced by pressure in these non-f-electron-containing $\mathrm{Ca}_{100-\mathrm{x}} \mathrm{Al}_{\mathrm{x}}$ $(x=20,27.3$ and 33.6 at.\%) metallic glasses. These results obtained clearly point out that the amorphous-to-amorphous configuration changes induced by pressure are not limited to $\mathrm{f}$ electron-containing metallic glasses. This will open a new vista which will trigger more theoretical and experimental investigations in this and many other MG systems.

\section{Methods}

$\mathrm{Ca}_{100-\mathrm{x}} \mathrm{Al}_{\mathrm{x}}(\mathrm{x}=20,27.3,33.6$ and 50 at.\%) MG ribbons with a thickness of about 35 $\mu \mathrm{m}$ and a width of about $3 \mathrm{~mm}$ were prepared with the single-roller melt-spinning method. In-situ high-pressure angle-dispersive XRD experiments with a wavelength of $0.6884 \AA$ and a focused beam size of about $5 \times 5 \mu \mathrm{m}^{2}$ were performed at the beamline 15U, Shanghai Synchrotron Radiation Facility (SSRF) in China. The $\mathrm{Ca}_{100-\mathrm{x}} \mathrm{Al}_{\mathrm{x}}\left(\mathrm{x}=20,27.3,33.6\right.$ and 50 at.\%) MGs were cut into about $40 \times 40 \times 35 \mu \mathrm{m}^{3}$ chips, and then loaded into a Mao-type symmetric diamond anvil cell. The sample chamber was about $150 \mu \mathrm{m}$ in diameter drilled in a T301 stainless steel gasket. Silicone oil was used as pressure-transmitting medium while for pressure calibration Au powders were dispersed inside. The pressure applied to the sample was calculated from the lattice constant of Au using the equation of state of $\mathrm{Au}^{36}$. The silicone oil as a pressure medium used in this work could remain hydrostatic up to about $15 \mathrm{GPa}$. The pressures when the anomalous changes detected in this work for $\mathrm{Ca}_{80} \mathrm{Al}_{20}$, $\mathrm{Ca}_{72.7} \mathrm{Al}_{27.3}$, and $\mathrm{Ca}_{66.4} \mathrm{Al}_{33.6} \mathrm{MGs}$ were at about $18 \mathrm{GPa}, 15.5 \mathrm{GPa}$ and $7.5 \mathrm{GPa}$, respectively. The changes detected below and above $15 \mathrm{GPa}$ indicate that these anomalous changes detected here are not strongly affected by the non-hydrostatic pressure caused by the pressure medium. Each XRD pattern was collected for about 5 seconds at a given pressure using a Mar-165 CCD detector and then integrated with the FIT2D program ${ }^{37}$. Ca $K$-edge XANES measurements for $\mathrm{Ca}_{100-\mathrm{x}} \mathrm{Al}_{\mathrm{x}}(\mathrm{x}=20,27.3$, 33.6 and 50 at.\%) MGs were carried out in vacuum at the beamline $4 \mathrm{~B} 7 \mathrm{~A}$, Beijing Synchrotron Radiation Facility in China.

Theoretical Ca K-edge XANES for fcc-Ca at $19.8 \mathrm{GPa}$, for bcc-Ca at $19.9 \mathrm{GPa}$, and for $\mathrm{Ca}_{13} \mathrm{Al}_{14}$ and $\mathrm{Ca}_{8} \mathrm{Al}_{3}$ alloys at ambient pressure were carried out in the framework of the multiple-scattering (MS) theory ${ }^{38,39}$ using the FEFF $8.2 \operatorname{code}^{40}$. The cluster for the simulations was generated by the ATOMS package ${ }^{41}$ using their respective lattice parameters ${ }^{23,24}$. In calculations, the Hedin-Lundqvist exchange-correlation potential was chosen ${ }^{42}$. A cluster of 43 atoms (for fcc-Ca) and 27 atoms (for bcc-Ca) were used in all MS calculations to obtain accurate self-consistent field (SCF), and the full MS calculation converges using a cluster up to $6 \AA$. It should be mentioned that Ca atoms in $\mathrm{Ca}_{13} \mathrm{Al}_{14}$ and $\mathrm{Ca}_{8} \mathrm{Al}_{3}$ alloys occupy more than one site, i.e. 5 sites for $\mathrm{Ca}_{8} \mathrm{Al}_{3}$ and 16 sites for $\mathrm{Ca}_{13} \mathrm{Al}_{14}$. A XANES spectrum contains all local structural information of different sites of the absorbed atomic species. Therefore, in the calculation for both $\mathrm{Ca}_{13} \mathrm{Al}_{14}$ and $\mathrm{Ca}_{8} \mathrm{Al}_{3}$ alloys we calculated the XANES spectrum of each individual site and then did a weighted superposition of all occupied sites. First-principles calculations based on density functional theory (DFT) in generalized gradient approximation (GGA) with the Perdew-Burke-Ernzerhof (PBE) functional ${ }^{43}$ were used to optimize crystal structures and obtain ground-state properties. Self-consistent calculations were performed using a plane-wave basis set limited by a cutoff energy of $400 \mathrm{eV}$ implemented in the Vienna ab initio simulation package (VASP). The projector augmented wave potentials ${ }^{44}$ and Monkhorst-Pack k-points sampling ${ }^{45}$ were employed. The convergence for the number of $\mathrm{k}$ points was tested to ensure that the total energy was converged within at least $0.01 \mathrm{eV} /$ atom.

1. Mishima, O., Calvert, L. D. \& Whalley, E. 'Melting Ice' I at $77 \mathrm{~K}$ and 10 kbar: a new method of making amorphous solids. Nature 310, 393-395 (1984).

2. Mishima, $\mathrm{O}$. Reversible first-order transition between two $\mathrm{H}_{2} \mathrm{O}$ amorphs at $\sim 0.2 \mathrm{GPa}$ and $\sim 135 \mathrm{~K}$. J Chem Phys 100, 5910-5912 (1994).

3. Stanley, H. E. \& Mishima, O. The relationship between liquid, supercooled and glassy water. Nature 396, 329-335 (1998).

4. Mishima, O. Liquid-liquid critical point in heavy water. Phys Rev Lett 85, 334-336 (2000).

5. Poole, P. H., Sciortino, F., Essmann, U. \& Stanley, H. E. Phase-Behavior of Metastable Water. Nature 360, 324-328 (1992).

6. Kingma, K. J., Meade, C., Hemley, R. J., Mao, H. K. \& Veblen, D. R. Microstructural Observations of Alpha-Quartz Amorphization. Science 259, 666669 (1993).

7. Lacks, D. J. First-order amorphous-amorphous transformation in silica. Phys Rev Lett 84, 4629-4632 (2000).

8. Sastry, S. \& Angell, C. A. Liquid-liquid phase transition in supercooled silicon. Nat Mater 2, 739-743 (2003).

9. Vanthiel, M. \& Ree, F. H. High-Pressure Liquid-Liquid Phase-Change in Carbon. Phys Rev B 48, 3591-3599 (1993).

10. Loerting, T., Brazhkin, V. V. \& Morishita, T. in Advances in Chemical Physics, Vol 143 (ed Rice, S. A. ) 29-82 (Wiley-Blackwell, 2009).

11. Zeng, Q. S. et al. Anomalous compression behavior in lanthanum/cerium-based metallic glass under high pressure. P Natl Acad Sci USA 104, 13565-13568 (2007).

12. Sheng, H. W. et al. Polyamorphism in a metallic glass. Nat Mater 6, 192-197 (2007).

13. Yavari, A. R. The changing faces of disorder. Nat Mater 6, 181-182 (2007).

14. Zeng, Q. S. et al. Origin of Pressure-Induced Polyamorphism in $\mathrm{Ce}_{75} \mathrm{Al}_{25}$ Metallic Glass. Phys Rev Lett 104, 105702 (2010).

15. Zeng, Q. S. et al. Properties of polyamorphous $\mathrm{Ce}_{75} \mathrm{Al}_{25}$ metallic glasses. Phys Rev B 82, 054111 (2010).

16. Zeng, Q. S. et al. Low-density to high-density transition in $\mathrm{Ce}_{75} \mathrm{Al}_{23} \mathrm{Si}_{2}$ metallic glass. J Phys-Condens Mat 22, 375404 (2010).

17. Zeng, Q. S. et al. Low temperature transport properties of Ce-Al metallic glasses. J Appl Phys 109, 113716 (2011).

18. Zeng, Q. S. et al. Substitutional alloy of Ce and Al. P Natl Acad Sci USA 106, 25152518 (2009). 
19. Zeng, Q. S. et al. Long-Range Topological Order in Metallic Glass. Science 332, 1404-1406 (2011)

20. Jiang, J. Z., Roseker, W., Sikorski, M., Cao, Q. P. \& Xu, F. Pressure effect of glass transition temperature in $\mathrm{Zr}_{46.8} \mathrm{Ti}_{8.2} \mathrm{Cu}_{7.5} \mathrm{Ni}_{10} \mathrm{Be}_{27.5}$ bulk metallic glass. Appl Phys Lett 84, 1871-1873 (2004)

21. Yavari, A. R. et al. Excess free volume in metallic glasses measured by X-ray diffraction. Acta Mater 53, 1611-1619 (2005).

22. Ma, D., Stoica, A. D. \& Wang, X. L. Power-law scaling and fractal nature of medium-range order in metallic glasses. Nat Mater 8, 30-34 (2009).

23. Gu, Q. F., Krauss, G., Grin, Y. \& Steurer, W. Experimental confirmation of the stability and chemical bonding analysis of the high-pressure phases Ca-I, II, and III at pressures up to 52 GPa. Phys Rev B 79, 134121 (2009).

24. Huang, B. Q. \& Corbett, J. D. Two new binary calcium-aluminum compounds: $\mathrm{Ca}_{13} \mathrm{Al}_{14}$, with a novel two-dimensional aluminum network, and $\mathrm{Ca}_{8} \mathrm{Al}_{3}$, an $\mathrm{Fe}_{3} \mathrm{Al}$-type analogue. Inorg. Chem. 37, 5827-5833 (1998).

25. Olijnyk, H. \& Holzapfel, W. B. Phase-Transitions in Alkaline-Earth Metals under Pressure. Phys Lett A 100, 191-194 (1984).

26. Ahuja, R., Eriksson, O., Wills, J. M. \& Johansson, B. Theoretical Confirmation of the High-Pressure Simple Cubic Phase in Calcium. Phys Rev Lett 75, 3473-3476 (1995).

27. Yabuuchi, T., Nakamoto, Y., Shimizu, K. \& Kikegawa, T. New high-pressure phase of calcium. J Phys Soc Jpn 74, 2391-2392 (2005)

28. Maksimov, E. G., Magnitskaya, M. V. \& Fortov, V. E. Non-simple behavior of simple metals at high pressure. Phys. Usp. 48, 761-780 (2005).

29. Oganov, A. et al. Exotic behavior and crystal structures of calcium under pressure. P Natl Acad Sci USA 107, 7646-7651 (2010).

30. Teweldeberhan, A. M., Dobois, J. L. \& Bonev, S. A. High-Pressure Phases of Calcium: Density- Functional Theory and Diffusion Quantum Monte Carlo Approach. Phys Rev Lett 105, 235503 (2010).

31. Maroni, B. et al. Pressure Effects in the Isoelectronic $\mathrm{REFe}_{0.85} \mathrm{Ir}_{0.15} \mathrm{AsO}$ System. $J$ Am Chem Soc 133, 3252-3255 (2011).

32. Sergeenkov, S. Influence of chemical pressure effects on nonlinear thermal conductivity of intrinsically granular superconductors. J Appl Phys 102, 066104 (2007).

33. Lue, C. S., Liu, R. F., Song, M. Y., Wu, K. K. \& Kuo, Y. K. Chemical pressure effect on the transport and electronic band structure of $\mathrm{Fe}_{2} \mathrm{~V}_{1-\mathrm{x}} \mathrm{Nb}_{\mathrm{x}} \mathrm{Al}$. Phys Rev B 78, 165117 (2008).

34. Ru, N. et al. Effect of chemical pressure on the charge density wave transition in rare-earth tritellurides $\mathrm{RTe}_{3}$. Phys Rev B 77, 035114 (2008).

35. Horii, T. et al. Chemical pressure effect in the heavy fermion compound $\mathrm{Ce}_{1-\mathrm{y}} \mathrm{La}_{\mathrm{y}}\left(\mathrm{Ru}_{0.85} \mathrm{Rh}_{0.15}\right)_{2} \mathrm{Si}_{2}$. J Magn Magn Mater 310, 313-315 (2007)

36. Anderson, O. L., Isaak, D. G. \& Yamamoto, S. Anharmonicity and the Equation of State for Gold. J Appl Phys 65, 1534-1543 (1989).

37. Hammersley, A. P., Svensson, S. O., Hanfland, M., Fitch, A. N. \& Hausermann, D. Two-dimensional detector software: From real detector to idealised image or twotheta scan. High Pressure Res 14, 235-248 (1996).

38. Lee, P. A. \& Pendry, J. B. Theory of Extended X-Ray Absorption Fine-Structure. Phys Rev B 11, 2795-2811 (1975).
39. Natoli, C. R., Benfatto, M., Brouder, C., Lopez, M. F. R. \& Foulis, D. L. Multichannel Multiple-Scattering Theory with General Potentials. Phys Rev B 42, 1944-1968 (1990).

40. Ankudinov, A. L., Ravel, B., Rehr, J. J. \& Conradson, S. D. Real-space multiplescattering calculation and interpretation of $\mathrm{x}$-ray-absorption near-edge structure. Phys Rev B 58, 7565-7576 (1998).

41. Ravel, B. ATOMS: crystallography for the X-ray absorption spectroscopist. J Synchrotron Radiat 8, 314-316 (2001)

42. Tyson, T. A., Hodgson, K. O., Natoli, C. R. \& Benfatto, M. General MultipleScattering Scheme for the Computation and Interpretation of X-Ray-Absorption Fine-Structure in Atomic Clusters with Applications to $\mathrm{SF}_{6}, \mathrm{GeCl}_{4}$, and $\mathrm{Br}_{2}$ Molecules. Phys Rev B 46, 5997-6019 (1992).

43. Perdew, J. P., Burke, K. \& Ernzerhof, M. Generalized gradient approximation made simple. Phys Rev Lett 77, 3865-3868 (1996).

44. Blochl, P. E. Projector Augmented-Wave Method. Phys Rev B 50, 17953-17979 (1994).

45. Monkhorst, H. J. \& Pack, J. D. Special Points for Brillouin-Zone Integrations. Phys Rev B 13, 5188-5192 (1976).

\section{Acknowledgments}

The authors would like to thank Shanghai Synchrotron Radiation Source, Hefei and Beijing Synchrotron Radiation facility for use of the synchrotron radiation facilities. Financial support from the National Key Basic Research Program of China (2012CB825700), the National Natural Science Foundation of China (Grants Nos. 50920105101, 51050110136, 51071141, 10979002 and 10904127), the Knowledge Innovation Program of the Chinese Academy of Sciences (KJCX2-YW-N42), the Ministry of Education of China, Zhejiang University-Helmholtz cooperation fund and the Department of Science and Technology of Zhejiang province is gratefully acknowledged. The magic supercomputer in Shanghai Supercomputer Center (SSC) is also acknowledged for supporting calculations.

\section{Author contributions}

JZJ designed research; HBL, YKF, QSZ, KY, XHY, LZ, YDZ, XDW, QPC performed experiments; HBL, LYH, WSC, ZYW, TDH, RA, JZJ analyzed data; JZJ wrote the paper.

\section{Additional information}

Supplementary information accompanies this paper at http://www.nature.com/ scientificreports

Competing financial interests: The authors declare no competing financial interests.

License: This work is licensed under a Creative Commons

Attribution-NonCommercial-ShareAlike 3.0 Unported License. To view a copy of this license, visit http://creativecommons.org/licenses/by-nc-sa/3.0/

How to cite this article: Lou, H.B. et al. Pressure-induced amorphous-to-amorphous configuration change in Ca-Al metallic glasses. Sci. Rep. 2, 376; DOI:10.1038/srep00376 (2012). 